\title{
EFECTO DE APLICAR INERCIA VIRTUAL EN LOS PARQUES EÓlICOS Los Cocos, LARIMAR I Y LARIMAR II en República Dominicana
}

\section{Effect of applying virtual inertia in the wind farms: Los Cocos, Larimar I and Larimar II in Dominican Republic}

\author{
Miguel Euclides Aybar Mejía a, Elvin Arnaldo Jiménez Matos ${ }^{\mathrm{b}}$, \\ Carlos Ángel Burgo ${ }^{c}$, Radhy Andrés Alcántara ${ }^{\mathrm{d}}$, Moisés Ismael \\ Blanco Sánchez ${ }^{e}$, Ryan Cabral Soto f, David Quezada Peña ${ }^{g}$ \\ y Carolyn Rodriguez Ramirez ${ }^{\mathrm{h}}$
}

Recibido: 17/01/20 • Aprobado: 13/03/20

Cómo citar: Aybar Mejía, M. E., Jiménez Matos, E. A., Burgo, C. Ángel, Alcántara, R. A., Blanco Sánchez, M. I., Cabral Soto, R., Quezada Peña, D., \& Rodríguez Ramírez, C. (2020). Efecto de aplicar inercia virtual en los parques eólicos: Los Cocos, Larimar I y Larimar II en República Dominicana. Ciencia, Ingenierías y Aplicaciones, 3(1), 31-54. Doi: https://doi.org/10.22206/cyap.2020.v3i1.pp31-54

\section{Resumen}

Con el creciente uso de energía eólica surge la necesidad de modificar los sistemas eléctricos de potencia, debido a la falta de inercia que caracteriza la generación renovable. Se plantea emular la respuesta inercial de aerogeneradores para mitigar estos efectos ocasionados por una configuración que le impide reaccionar ante fallas ocurridas en la red eléctrica. Para validación de lo propuesto se estudia la aplicación de inercia virtual en los parques eólicos Los Cocos, Larimar 1 y Larimar 2, que representan el mayor porcentaje de generación eólica de la República Dominicana. Para analizar el efecto de la configuración propuesta en la estabilidad del Sistema Eléctrico Nacional Interconectado se utilizó el software Power Factory DIgSILENT,

\footnotetext{
a Ingeniería Eléctrica, Instituto Tecnológico de Santo Domingo (INTEC), República Dominicana Correo-e: miguel.aybar@intec.edu.do

b EgeHaina, Departamento de protecciones. Ingeniería Eléctrica, Instituto Tecnológico de Santo Domingo (INTEC), República Dominicana. Correo-e: elvin.jimenez@intec.edu.do

c EgeHaina, Departamento de Protecciones, República Dominicana.

Correo-e: burgoc@egehaina.com

*Ingeniería Eléctrica, Instituto Tecnológico de Santo Domingo (INTEC)

d Correo-e: radhyalcantara@gmail.com, e Correo-e: moizezblanco@gmail.com

${ }^{f}$ Correo-e: ryancabral0113@gmail.com, ${ }^{\mathrm{g}}$ Correo-e: david.quezada.p@gmail.com

h Correo-e: carolynrr20@gmail.com
}

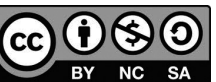

Esta obra está bajo licencia internacional Creative Commons Atribución-NoComercial-CompartirIgual 4.0 Internacional 
donde se hacen simulaciones bajo distintos parámetros de carga para obtener las curvas de variación de la frecuencia eléctrica frente a distintos valores de la constante de inercia al ocurrir una falla por desconexión de una central generadora. Con el fin de aplicar la inercia virtual en los aerogeneradores se utiliza la electrónica de potencia para realizar la inyección de potencia activa a la red eléctrica cuando ocurre una falla en el sistema eléctrico. Se desarrolla un modelo dinámico con base en el control ROCOF (Rate of Change of Frequency) cuyo objetivo es inyectar potencia activa al sistema dependiendo de la variación de frecuencia. Realizando las simulaciones se comprueba que aplicando inercia virtual se obtiene una mayor estabilidad en el sistema eléctrico y menos probabilidad de un posible colapso del sistema eléctrico.

Palabras clave: aerogenerador; generador de inducción doblemente alimentado (DFIG); estabilidad del sistema de potencia; full converter, DIgSILENT; inercia virtual; respuesta en inercia; generador de inducción.

\begin{abstract}
With the increasing use of wind energy, the need arises to modify electrical power systems, due to the lack of inertia that characterizes renewable generation. It is proposed to emulate the inertial response of wind turbines to mitigate these effects caused by a configuration that prevents it from reacting to failures in the electrical network. To validate what is proposed, the application of virtual inertia is studied in the Los Cocos, Larimar 1, and Larimar 2 wind farms, which represent the highest percentage of wind generation in the Dominican Republic. To analyze the effect of the proposed configuration on the stability of the Interconnected National Electric System, the Power Factory DIgSILENT software was used, where simulations are carried out under different load parameters to obtain the curves of variation of the electrical frequency against different values of the constant inertia when a fault occurs due to disconnection of a generating plant. To apply virtual inertia to wind turbines, power electronics are used to inject active power into the electrical grid when a fault occurs in the electrical system. A dynamic model is developed based on the ROCOF (Rate of Change of Frequency) control whose objective is to inject active power into the system depending on the frequency variation. Performing simulations, it is found that applying virtual inertia greater stability is obtained in the electrical system and less chance of a possible collapse of the electricity system.
\end{abstract}

Keywords: Wind turbine; double-powered induction generator (DFIG); power system stability; full converter; DIgSILENT; virtual inertia; inertia response; induction generator. 


\section{Introducción}

La estabilidad es la capacidad de un sistema eléctrico de mantenerse en equilibrio tras ocurrir una perturbación transitoria en un sistema de potencia (Gómez \& Rivera, 2018). Dichas irregularidades están supuestas a pasar por alto en la calidad de la energía suministrada. Desde el enfoque de estabilidad la respuesta en inercia no es más que la garantía del control de confiabilidad, seguridad y calidad en el sistema eléctrico.

La respuesta inercial es una característica de los generadores síncronos en los sistemas de potencia, ya que gracias a la energía cinética almacenada en sus partes rotatorias son los primeros en actuar cuando ocurren contingencias que incurran en un desequilibrio entre generación y demanda, traducidos en cambios en la frecuencia resultante (Marabolí Muñoz, 2019). Es por tanto que la respuesta en inercia virtual de un sistema eléctrico de potencia significa la oposición en forma de cualquier intercambio de energía de máquinas sincronizadas para disminuir los cambios o desequilibrio de potencia en la generación y demanda, reflejado en la frecuencia resultante (Tielens, Van Hertem, \& Reviews, 2016).

El generador de inducción doblemente alimentado DFIG es una máquina de inducción que posee un rotor bobinado con su circuito de rotor conectado a la red a través de un sistema de convertidores de electrónica de potencia, mientras que el estator es conectado directamente a la red (Viltre, Lesyani, Hernández Amador \& Aybar Mejia, 2019). En los últimos ańos ha tenido un crecimiento significativo debido a la alta eficiencia que presentan para distintos niveles de velocidad. Sin embargo, estos generadores eléctricos tienen la desventaja de que su respuesta en inercia es deficiente debido a los convertidores auto conmutados de corriente alterna (Sánchez \& Alonso, 2016).

El Full Converter es un tipo de generador eléctrico cuyas características constructivas hacen que su sistema mecánico se desacople del sistema de potencia eléctrico (Riva, Bada, \& Alvez, 2016). La conversión de potencia es realizada por un convertidor del lado de la máquina (MSC, 
Machine-Side Converter) y un convertidor del lado de la red (GSC, Grid-Side Converter).

Basándose en la matriz energética de la República Dominicana, la energía eólica figura como la segunda mayor fuente primaria de energía renovable para la producción eléctrica según los datos recuperados del Informe Mensual de Operación Real Julio del OC, 2019, lo que significa que cualquier aparición, regulación o alternación que ocurra en dicha tecnología será directamente proporcional a un cambio en la estabilidad del sistema eléctrico de potencia.

El objetivo de este proyecto es analizar el impacto de aplicar inercia virtual como herramienta de control para aumentar la estabilidad del Sistema Eléctrico Nacional Interconectado (SENI) en los parques eólicos Los cocos, Larimar I y Larimar II. Se realizarán estudios basados en simulaciones que permiten estudiar los efectos producidos en el comportamiento del SENI, en conjunto con los posibles beneficios que resultan de aplicar la tecnología de inercia virtual en los aerogeneradores de Los Cocos, Larimar I y Larimar II.

\section{Metodología}

La estrategia de trabajo utilizada para esta investigación se basó en la recolección de datos, análisis de información, puesta en práctica y análisis de resultados. El primer paso consistió en una revisión profunda del estado del arte, para determinar, a nivel mundial, los avances de otros autores que tratan el tema de inercia virtual en aerogeneradores, y así tener una idea de cómo se está abordando dicha problemática actualmente en otros países, cuáles son sus parámetros de análisis, las herramientas que utilizan y el alcance que le dan a cada una de sus investigaciones.

Luego, se eligió un método para aplicar inercia virtual, el método seleccionado se desarrolló en el artículo Virtual Inertia: Current Trends and Future Directions (Tamrakar et al., 2017). En este se explican distintos métodos para aplicar inercia virtual en aerogeneradores (ver tabla 1). 
De entre ellos se seleccionó el que se enfoca en la caída de la frecuencia eléctrica. Se escogió este criterio debido a su similitud con el control de caída convencional de un generador síncrono.

La siguiente ecuación se utiliza para obtener la potencia activa que entregará el aerogenerador cuando se aplica inercia virtual

$$
\Delta P=2 H_{\text {syn }} * f_{\text {Syst }} * \frac{d f_{\text {syst }}}{d t}
$$

donde:

$\Delta \mathbf{P}$ : potencia activa a entregar

$\mathbf{H}_{\text {Syn }}$ : se refiere a la inercia sintética (Seg.)

$\mathbf{F}_{\text {Sist }}:$ representa la frecuencia del sistema eléctrico (P.U.)

$\frac{\boldsymbol{d} \boldsymbol{f}_{\boldsymbol{s y s t}}}{\boldsymbol{d} \boldsymbol{t}}$ : será la derivada de la frecuencia $(\mathrm{Hz} / \mathrm{s})$.

La ecuación 1 se utilizó para obtener la potencia eléctrica que inyectará el aerogenerador al momento de ocurrir una falla; la ecuación fue obtenida del artículo Activation Schemes of Synthetic Inertia Controller on Full Converter Wind Turbine (Type 4) (Gonzalez-Longatt, 2015).

El enfoque de caída de frecuencia $(R o C o F)$ fue el método seleccionado para utilizarse en los parques eólicos administrados por la Empresa de Generación Eléctrica Haina, ya que dicha empresa representa el mayor porcentaje de generación eólica de $175 \mathrm{MW}$ (47.94\%) de los 365,25 MW instalados en República Dominicana.

Las simulaciones se realizaron en el software DIgSILENT, el cual es un acrónimo creado a partir del inglés "Digital SImuLation and Eléctrica NeTworks". Se optó por utilizar este software debido a su fácil manejo, debido a que no presenta una gran dificultad a la hora de la simulación en comparación a software como PSS/E y simulink, posee una interfaz gráfica más amigable y varios modelos de referencia como se indica en la 
tabla 2. La base de datos del sistema eléctrico de la República Dominicana que se utilizó para realizar este proyecto fue obtenida del Organismo Coordinador del Sistema Eléctrico Nacional Interconectado (OC-SENI) para el día 16 de diciembre del año 2019.

Los generadores DFIG y full converters no aportan inercia debido a su condición de no estar conectados a la red directamente, y por esto no cuentan con un modelo para aplicar inercia virtual. De ahí que, para poner en funcionamiento la inercia virtual, se creó un modelo de control que deberá inyectar potencia activa en función al cambio instantáneo de frecuencia ocurrido por fallas por desconexión de una central en el SENI. El modelo de inercia virtual se explica en el apartado de simulación.

Es preciso resaltar que las simulaciones de inercia virtual solo se activarán en momentos de falla por desconexión según los escenarios definidos en este estudio. La inercia virtual se aplica a un solo bloque que representa la potencia conjunta de todos los parques, los aerogeneradores están conectados a la red mediante electrónica de potencia, más específicamente mediante un inversor back to back que se encarga de inyectar a la red la potencia eléctrica generada por el aerogenerador y, al mismo tiempo, de aislar la máquina de cualquier falla que ocurra en la red. Según los datos proporcionados por EGEHaina, los inversores back to back se encuentran sobredimensionados a la potencia nominal de los aerogeneradores, por lo que el concepto de aplicar inercia virtual tratado en este proyecto se aplica dentro de la programación del inversor como alternativa a mantener un porcentaje de la potencia total de los parques como reserva para inercia. En este caso se ha seleccionado un $10 \%$ de la potencia total de los parques, ya que representa un porcentaje dentro del margen del sobredimensionamiento del back to back, que resulta significativo al momento de realizar las simulaciones y que el mismo no afectará la vida útil de estos si se aplicara en sus inversores. Es importante destacar que este $10 \%$ representa un valor aproximado al que los inversores podrían entregar en realidad, para seleccionar un porcentaje de potencia más exacto se debe de realizar un estudio técnico independiente a los inversores. 
Tabla 1. Técnicas de control de inercia virtual (Tamrakar et al., 2017).

\begin{tabular}{|c|c|c|}
\hline Método de Control & Caracteristicas & Desventajas \\
\hline $\begin{array}{l}\text { Modelo basado } \\
\text { en generadores } \\
\text { síncronos }\end{array}$ & $\begin{array}{l}\text { - } \begin{array}{l}\text { Replicación precisa de la } \\
\text { dinámica de generadores } \\
\text { síncronos. }\end{array} \\
\text { - No requiere de derivada } \\
\text { de la frecuencia. } \\
\text { - Bucle de fase bloqueada } \\
\text { (PLL) solo para } \\
\text { sincronización. }\end{array}$ & $\begin{array}{l}\text { - Inestabilidad numérica. } \\
\text { Implementación de } \\
\text { fuente de voltaje; } \\
\text { no protección por } \\
\text { sobrecorriente. }\end{array}$ \\
\hline $\begin{array}{l}\text { Modelo basado } \\
\text { en la ecuación de } \\
\text { oscilación (Swing } \\
\text { Equation) }\end{array}$ & $\begin{array}{l}\text { - Más simple que el } \\
\text { basado en generadores } \\
\text { síncronos. } \\
\text { - } \quad \begin{array}{l}\text { No requiere de derivada } \\
\text { de la frecuencia. }\end{array} \\
\text { Bucle de fase bloqueada } \\
\text { o Phase Locked Loop } \\
\text { (PLL) solo para } \\
\text { sincronización. }\end{array}$ & $\begin{array}{l}\text { - Oscilaciones de } \\
\text { frecuencia y potencia. } \\
\text { Implementación de } \\
\text { fuente de voltaje; } \\
\text { no protección por } \\
\text { sobrecorriente. }\end{array}$ \\
\hline $\begin{array}{l}\text { Modelo basado } \\
\text { en respuesta } \\
\text { frecuencia- } \\
\text { potencia }\end{array}$ & $\begin{array}{l}\text { Implementación sencilla } \\
\text { y directa. } \\
\text { Implementación de } \\
\text { fuente de corriente; } \\
\text { con protección por } \\
\text { sobrecorriente. }\end{array}$ & $\begin{array}{l}\text { - Inestabilidad debida al } \\
\text { bucle de fase bloqueada } \\
\text { o Phase Locked Loop } \\
\text { (PLL). } \\
\text { - Requiere de derivada de } \\
\text { la frecuencia; sistema } \\
\text { susceptible al ruido. }\end{array}$ \\
\hline $\begin{array}{l}\text { Enfoque basado } \\
\text { en la caída }\end{array}$ & $\begin{array}{l}\text { - Sin comunicación. } \\
\text { - Conceptos similares } \\
\text { al control de caídas } \\
\text { tradicional de los } \\
\text { generadores síncronos. }\end{array}$ & $\begin{array}{l}\text { - } \begin{array}{l}\text { Respuesta lenta y } \\
\text { transitoria. }\end{array} \\
\text { - Transferencia incorrecta } \\
\text { y transitoria de potencia } \\
\text { activa. }\end{array}$ \\
\hline
\end{tabular}


Tabla 2. Herramientas de simulación (Deepak, Vijayakumari \& Mohanrajan, 2017; DIgSILENT, 2019; Gonzalez-Longatt \& Energy, 2016; Khaleghi, Alamuti, Li, Taylor \& Bradley, 2014; Nuñez, Cepeda $\&$ Salazar, 2015; SIEMENS, 2019)

\begin{tabular}{lccccc} 
Software & $\begin{array}{c}\text { Tiempo de } \\
\text { respuesta }\end{array}$ & $\begin{array}{c}\text { Modelos } \\
\text { de } \\
\text { referencia }\end{array}$ & $\begin{array}{c}\text { Lenguaje de } \\
\text { programación }\end{array}$ & $\begin{array}{c}\text { Interfaz } \\
\text { gráfica }\end{array}$ & $\begin{array}{c}\text { Dificultad } \\
\text { de } \\
\text { simulación }\end{array}$ \\
\hline Simulink & \pm & + & - & + & \pm \\
DIgSILENT & - & + & + & + & + \\
PSSE & + & - & + & - & - \\
\hline
\end{tabular}

Nota: + significa excelente, \pm significa bueno, - significa normal.

\section{Variables}

Para realizar el análisis del impacto de la implementación de inercia virtual en los parques eólicos Los Cocos, Larimar I y Larimar II, es importante poder estimar su comportamiento al estar integrado al Sistema Eléctrico Nacional Interconectado (SENI). Por este motivo se necesita realizar simulaciones de los aerogeneradores luego de agregarle los bloques de inercia virtual y a su vez agregarlo al SENI.

Las variables que se tomaron en consideración para este estudio son:

- Derivada de frecuencia

- Frecuencia

- Inercia

- Potencia activa

- Tiempo 


\section{Simulación}

Para la fase de simulación se establecieron dos eventos: el primero, la salida de uno de los generadores de la central termoeléctrica Punta Catalina 1 de 377 MW, cuyo objetivo es ver el comportamiento de la frecuencia en el SENI con inercia virtual aplicada en los aerogeneradores, el segundo, la salida de la generadora en base a carbón ITABO 1 con 125 MW. Cada uno de estos eventos se dividirá en tres categorías, que son los periodos de demanda del sistema eléctrico de República Dominicana, y estos se subdividen en seis subcategorías más. Las categorías están basadas en lo que son los periodos de demanda del sistema eléctrico de República Dominicana, las horas en que estos ocurren, y las subcategorías son los distintos tiempos de inercia aplicados en los parques eólicos. Para las simulaciones se escogieron tres periodos distintos que representan la baja, media y alta demanda, los cuales se establecerán a continuación:

- P04: es el período de baja demanda en el SENI y corresponde a las horas 3:00 a. m. - 4:00 a. m.

- P12: período que corresponde a la demanda media en el SENI este corresponde a las horas 11:00 a. m. - 12:00 p. m.

- P21: este período es de alta demanda en el SENI y se establece a las horas 20:00 p. m. - 21:00 p. m.

Las subcategorías que son los tiempos aplicados a los parques eólicos son:

- H0: no se está aplicando inercia virtual en los parques eólicos mencionados.

- H1: se utiliza una constante de inercia de 1 segundo.

- H2: se utiliza una constante de inercia de 2 segundo.

- H3: se utiliza una constante de inercia de 3 segundo.

- H4: se utiliza una constante de inercia de 4 segundo.

- H5: se utiliza una constante de inercia de 5 segundo. 
Para cada período se monitoreará el comportamiento de dos gráficas principales: la frecuencia del sistema y la inyección de potencia activa de los parques eólicos en cuestión.

Tabla 3. Estructura de los escenarios de simulación

\begin{tabular}{|c|c|c|c|c|c|c|c|}
\hline Evento & Periodo & \multicolumn{6}{|c|}{ Escenarios } \\
\hline \multirow{3}{*}{$\begin{array}{c}\text { Disparo Punta Catalina } 1 \text { (377 } \\
\text { MW) }\end{array}$} & P04 & Ho & $\mathrm{H} 1$ & $\mathrm{H} 2$ & $\mathrm{H} 3$ & $\mathrm{H} 4$ & $\mathrm{H} 5$ \\
\hline & P12 & Ho & H1 & $\mathrm{H} 2$ & $\mathrm{H} 3$ & $\mathrm{H} 4$ & $\mathrm{H} 5$ \\
\hline & P21 & Ho & H1 & $\mathrm{H} 2$ & $\mathrm{H} 3$ & $\mathrm{H} 4$ & $\mathrm{H} 5$ \\
\hline \multirow{3}{*}{ Disparo Itabo 1 (125 MW) } & P04 & Ho & $\mathrm{H} 1$ & $\mathrm{H} 2$ & $\mathrm{H} 3$ & $\mathrm{H} 4$ & $\mathrm{H} 5$ \\
\hline & P12 & Ho & $\mathrm{H} 1$ & $\mathrm{H} 2$ & $\mathrm{H} 3$ & $\mathrm{H} 4$ & $\mathrm{H} 5$ \\
\hline & P21 & Ho & $\mathrm{H} 1$ & $\mathrm{H} 2$ & $\mathrm{H} 3$ & $\mathrm{H} 4$ & $\mathrm{H} 5$ \\
\hline
\end{tabular}

Para analizar el impacto de la aplicación de inercia virtual en los parques eólicos Los Cocos, Larimar I y Larimar II en la estabilidad del Sistema Eléctrico Nacional Interconectado, se realizaron simulaciones dinámicas en el software DIgSILENT. Fundamentalmente, la estructura y función objetivo de este análisis consiste en evaluar el comportamiento de la frecuencia del sistema y establecer una correlación con el aporte de potencia activa que inyectaría adicionalmente el conjunto de los parques eólicos, gracias a la participación de la inercia virtual. La figura 1 muestra la representación en DIgSILENT de los parques eólicos estudiados. Cabe destacar que aunque Los Cocos y Larimar corresponden a concesiones distintas, para fines de simulación no se modifican todos los bloques de los aerogeneradores, sino que como se marca en la figura 1 la inercia virtual se aplica a un solo bloque que representará la potencia conjunta de todos los parques. 

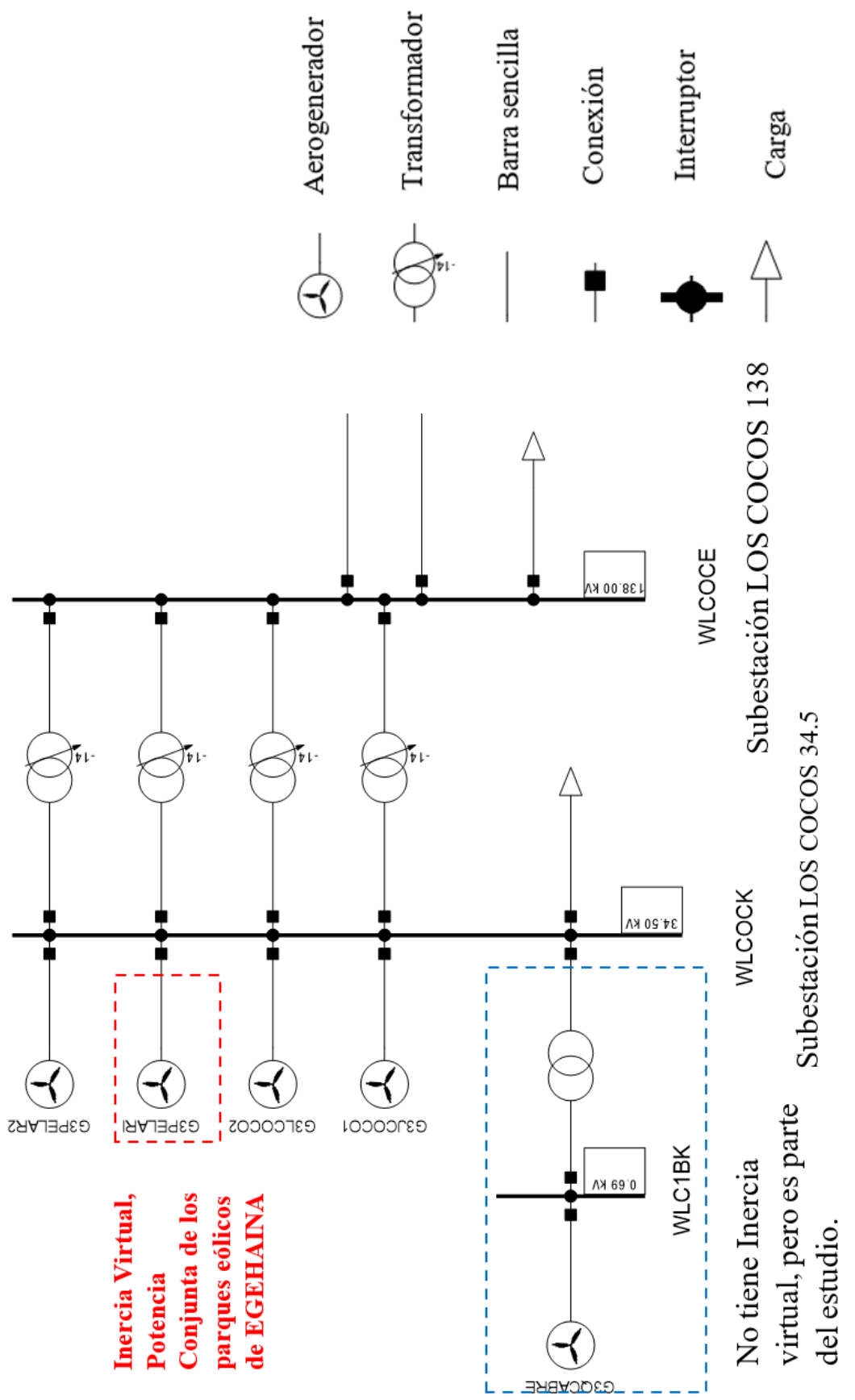

Figura 1. Vista en DIgSILENT de los parques eólicos evaluados 
El primer paso antes de realizar la simulación es determinar los parámetros con los que los aerogeneradores simularán inercia. El concepto de inercia virtual permite que un controlador tome la energía cinética de una masa giratoria y mediante un control bucle aumenta la producción de energía eléctrica durante un evento significativo de descenso de frecuencia. Con la ecuación 1 se define el comportamiento que debe presentar el aerogenerador al momento de ocurrir una desviación en la frecuencia eléctrica en el SENI (Gonzalez-Longatt, 2015). Para poder introducir la ecuación 1 dentro del aerogenerador y que este la reconozca como una instrucción se debe crear un bloque dentro del programa mediante el lenguaje de programación de DIgSILENT, o DSL por sus siglas en inglés, que le indique los parámetros que debe analizar y cómo debe actuar frente a los distintos valores que presenten los mismos.

En la figura 2 se presenta el diagrama de bloques en DSL a utilizar para la implementación de inercia virtual en los aerogeneradores de Los Cocos, Larimar I y Larimar II. Se utilizan dos bloques de medición, uno se encarga de medir el voltaje de la barra a la que se encuentra conectado el aerogenerador y el otro la frecuencia eléctrica del sistema, estos valores serán las entradas del bloque de aporte inercial ROCOF (Rate of Change of frecuency o Tasa de cambio de frecuencia) que contiene la ecuación 1 , pero con el lenguaje de programación propio de DIgSILENT, o DPL por sus siglas en inglés.

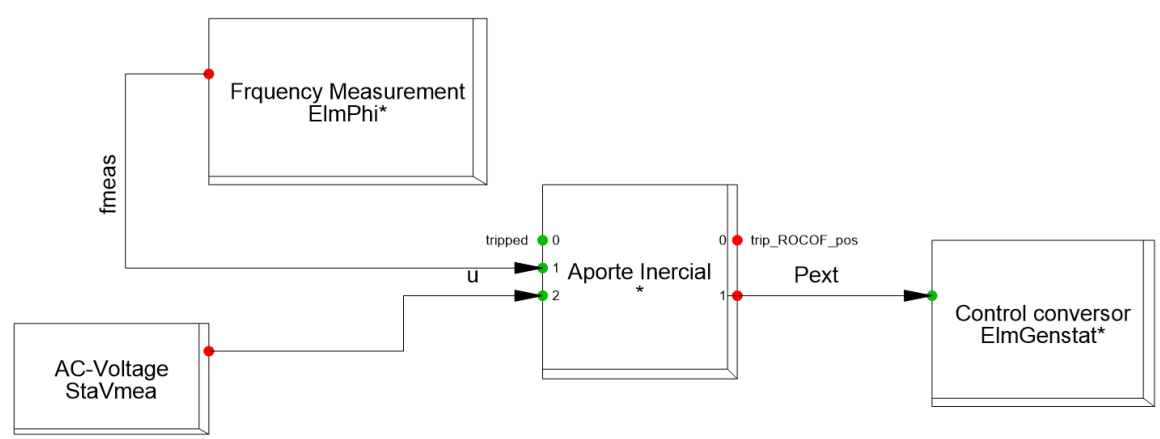

Figura 2. Diagrama de bloques DSL 


\section{Caracterización}

Con el fin de seleccionar la muestra del estudio se consideró la potencia total instalada en los parques eólicos según la matriz de generación energética de la República Dominicana mostrada en la tabla 4. También se analizó la disponibilidad de modelos para integrar la inercia virtual a los parques eólicos seleccionados y su integración al SENI con el uso del software DIgSILENT.

Tabla 4. Potencia instalada en los distintos parques eólicos

\begin{tabular}{|c|c|c|}
\hline Parque Eólico & Ubicación & Potencia (MW) \\
\hline Los Cocos I & Pedernales, Barahona & 25,2 \\
\hline Los Cocos II & Pedernales, Barahona & 52 \\
\hline Larimar I & Barahona & 49,5 \\
\hline Larimar II & Barahona & 48,3 \\
\hline \multicolumn{2}{|c|}{ Subtotal EGEHAINA } & $\mathbf{1 7 5}$ \\
\hline Quilvio Cabrera & Pedernales & 8,25 \\
\hline Matafongo & Peravia & 34 \\
\hline Los Guzmancitos & Puerto Plata & 48 \\
\hline Agua Clara & Monte Cristi & 50 \\
\hline Guanillo & Monte Cristi & 50 \\
\hline Total & & $\mathbf{3 6 5 , 2 5}$ \\
\hline
\end{tabular}

Los parques eólicos seleccionados para esta investigación se interconectan a una línea de transmisión de $138 \mathrm{kV}$. En la tabla 5 se muestran las características de los parques eólicos seleccionados. Dentro de estas características el tipo de aerogenerador presente es un factor determinante a la hora de realizar el estudio. 
Tabla 5. Datos de los aerogeneradores

\begin{tabular}{|c|c|c|c|c|}
\hline Parque eólico & $\begin{array}{c}\text { Puesta en } \\
\text { servicio }\end{array}$ & $\begin{array}{c}\text { Modelo de las } \\
\text { turbinas }\end{array}$ & $\begin{array}{c}\text { Tipo de } \\
\text { generador }\end{array}$ & $\begin{array}{c}\text { Potencia de } \\
\text { cada generador } \\
\text { (MW) }\end{array}$ \\
\hline Los Cocos I & 2010 & Vestas V90 & DFIG & 1,8 \\
\hline Los Cocos II & 2013 & Gamesa G97 & DFIG & 2 \\
\hline Larimar I & 2016 & Vestas V112 & $\begin{array}{c}\text { Full } \\
\text { Converter }\end{array}$ & 3,3 \\
\hline Larimar II & 2018 & Vestas V117 & $\begin{array}{c}\text { Full } \\
\text { Converter }\end{array}$ & 3,45 \\
\hline $\begin{array}{c}\text { Quilvio } \\
\text { Cabrera }\end{array}$ & 2011 & Vestas V82 & Asíncrono & 1,65 \\
\hline Matafongo & 2019 & Gamesa G97 & DFIG & 2 \\
\hline $\begin{array}{c}\text { Los } \\
\text { Guzmancitos }\end{array}$ & 2017 & Vestas V136 & Asíncrono & 3,45 \\
\hline Agua Clara & 2019 & Gamesa G114 & DFIG & 2 \\
\hline Guanillo & 2019 & Gamesa G114 & DFIG & 2 \\
\hline
\end{tabular}

\section{Análisis económico}

En el caso de la elaboración de un análisis económico, se tomarían en cuenta dos posibles escenarios:

- Evaluar la existencia de remuneraciones dentro del marco normativo que hace referencia a los estándares de regulación de frecuencia.

- Seleccionar un país que comparta características con el sector eléctrico dominicano pero que a su vez emule inercia virtual, de manera que permita extrapolar las remuneraciones que se obtienen por las regulaciones dadas en dicho país, e hipotéticamente plantear resultados con base en lo estudiado. 
El primer escenario quedaría descartado, puesto que según las regulaciones expuestas en la Ley 125-01, no hay un pago o beneficio por regulación de frecuencia a las centrales no convencionales. Teniendo en cuenta dichos artículos, queda estipulada en primera instancia la libertad de regulación por parte de los generadores de régimen especial, y que a su vez no son remunerados por ejecutar dicha acción.

En el segundo escenario, a nivel internacional, las remuneraciones que se obtienen a partir de la emulación de inercia virtual es por medio de reservas rotantes, un aspecto que no será abarcado para fines de respuestas, sino para fines informativos en el proyecto presentado.

\section{Análisis ambiental}

La generación de energía eléctrica mediante aerogeneradores no produce emisiones de gases de efecto invernadero, a diferencia de las plantas que trabajan con carbón que tienen una mayor emisión de $\mathrm{CO}_{2}$. La generación eólica puede ayudar a reducir la contaminación sustituyendo plantas de generación convencional (Balali, Nouri, Omrani, Nasiri, \& Otieno, 2017). Por ende, esto se puede utilizar para cumplir con el objetivo de reducir las emisiones de gases de efecto invernadero en un $25 \%$ para el año 2030 (Agencia Internacional de Energías Renovables [IRENA], 2017).

Según la Agencia de los Estados Unidos para el Desarrollo Internacional, la República Dominicana emitió 25,18 $\mathrm{MtCO}_{2}$ en el año 2018, presentando un aumento de 3,09\% con respecto a lo emitido en el 2013. El sector energía de la República Dominicana fue el que produjo más emisiones de gases de efecto invernadero con un $85,5 \%$, donde la generación de energía eléctrica representa alrededor del $50 \%$ de este sector (United States Agency for International Development, 2017). En la tabla 6 se presenta una lista de países con características geográficas parecidas a las de la República Dominicana y sus emisiones de gases de efecto invernadero en el año 2017. 
Tabla 6. Emisiones de CO2 por países (European Commission, 2018).

\begin{tabular}{|l|c|c|}
\hline \multicolumn{1}{|c|}{ País } & $\begin{array}{c}\text { Utilizan inercia } \\
\text { virtual }\end{array}$ & $\begin{array}{c}\text { Emisiones de } \mathrm{CO}_{2} \\
\left(\mathbf{M t C O}_{2} \boldsymbol{e}\right)\end{array}$ \\
\hline República Dominicana & No & 23,11 \\
\hline Costa Rica & Sí & 8,13 \\
\hline Eslovaquia & No & 37,85 \\
\hline Estonia & No & 17,88 \\
\hline Dinamarca & Sí & 33,57 \\
\hline Cuba & No & 31,27 \\
\hline Puerto Rico & No & 2,16 \\
\hline Jamaica & No & 7,54 \\
\hline
\end{tabular}

Para estimar la cantidad de $\mathrm{CO}_{2}$ emitido se utilizó el factor de emisiones global, el cual corresponde a 181 gramos de $\mathrm{CO}_{2}$ por cada $\mathrm{kWh}$ generado. Para el cálculo de las emisiones totales en un año se tomaron en cuenta los disparos de las centrales que podrían representar un mayor diferencial de frecuencia, todos estos datos se tomaron de las memorias de fallas del Organismo Coordinador del año 2018. Cabe destacar que se tomaron en cuenta 65 disparos y se asumió que los 17,5 MW serían un valor constante para cada disparo. Luego de realizar el análisis se concluyó que, si se aplica la inercia virtual en los parques eólicos Los Cocos, Larimar I y Larimar II la cantidad de $\mathrm{CO}_{2}$ que se dejará de emitir será de 7,2 toneladas de $\mathrm{CO}_{2}$ al año.

\section{Resultados}

A continuación, se presentan los resultados de las simulaciones detalladas en el acápite de Simulación, referentes a la aplicación de inercia virtual en los Parques Eólicos Los Cocos, y Larimar I y II, y sus efectos en la estabilidad del Sistema Eléctrico Nacional Interconectado. Para estas 
simulaciones se utilizaron los escenarios de demanda y generación del Programa Diario Definitivo del 16 de diciembre del 2019, emitido por el Organismo Coordinador - SENI.

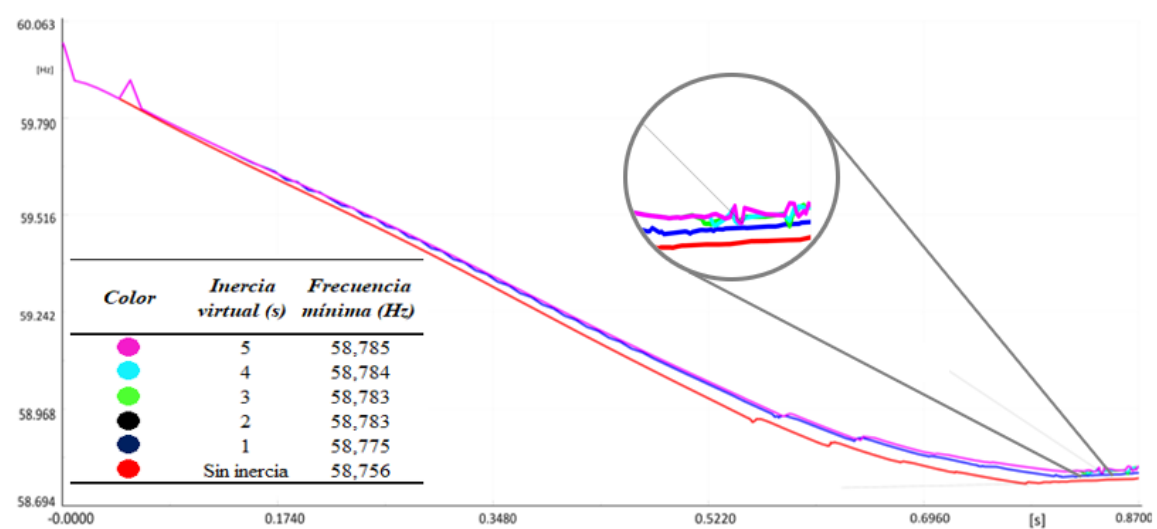

Figura 3. Evento Punta Catalina 1, escenario P04 - Gráfico resumen

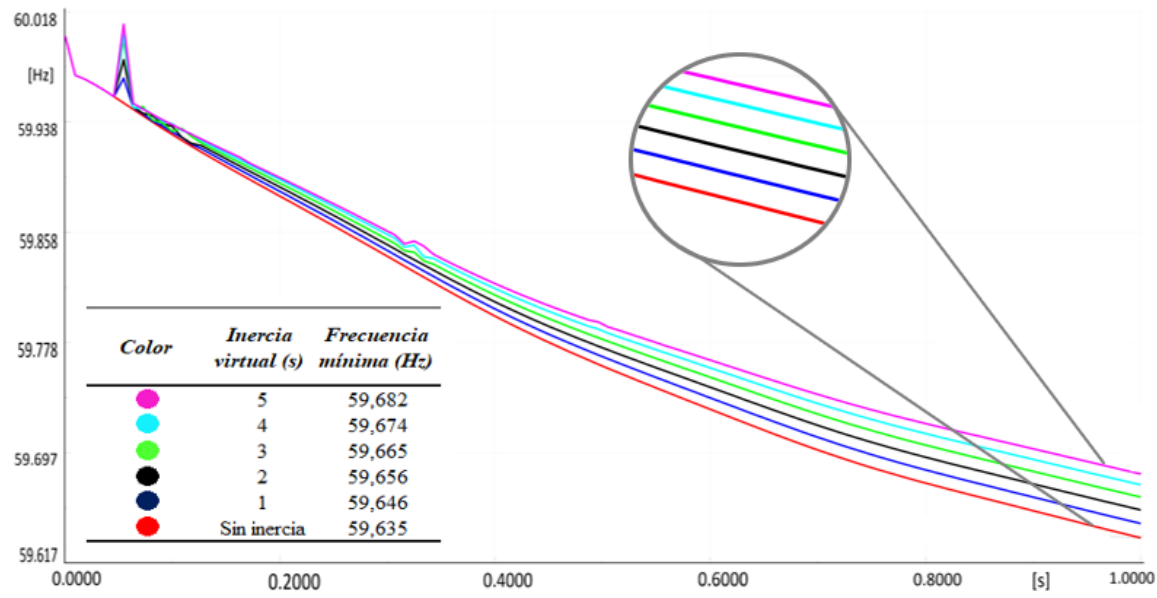

Figura 4. Evento Itabo 1, escenario P04 - Gráfico resumen 
Tabla 8. Disparo de la Central Termoeléctrica Punta Catalina 1 del periodo: 12 y 21 .

\begin{tabular}{ccc}
\hline Período & Inercia Virtual $(\mathbf{s})$ & Frecuencia Mínima $\mathbf{( H z )}$ \\
& Sin Inercia & 58,885 \\
P12 & 1 & 58,896 \\
& 2 & 58,901 \\
& 3 & 58,908 \\
& 4 & 58,899 \\
& 5 & 58,893 \\
\hline \multirow{4}{*}{ P21 } & Sin Inercia & 58,908 \\
& 1 & 58,923 \\
& 2 & 58,929 \\
& 3 & 58,930 \\
& 4 & 58,932 \\
5 & 58,932 \\
\hline
\end{tabular}

Tabla 9. Disparo de la Central Termoeléctrica Itabo 1 del periodo: 12 y 21.

\begin{tabular}{|c|c|c|}
\hline Período & Inercia Virtual (s) & Frecuencia Mínima (Hz) \\
\hline \multirow{4}{*}{ P12 } & Sin Inercia & 59,694 \\
\cline { 2 - 3 } & 1 & 59,701 \\
\cline { 2 - 3 } & 2 & 59,708 \\
\cline { 2 - 3 } & 3 & 59,714 \\
\cline { 2 - 3 } & 4 & 59,714 \\
\hline \multirow{4}{*}{ P21 } & 5 & 59,726 \\
\cline { 2 - 3 } & Sin Inercia & 59,694 \\
\cline { 2 - 3 } & 1 & 59,701 \\
\cline { 2 - 3 } & 2 & 59,708 \\
\cline { 2 - 3 } & 3 & 59,714 \\
\cline { 2 - 3 } & 4 & 59,720 \\
\hline
\end{tabular}




\section{Discusión de resultados}

Como se pudo observar en las simulaciones mostradas en la figura 3 y la figura 4, en los escenarios donde se encuentra activada la inercia virtual, el gráfico de la potencia activa inyectada por inercia responde al cambio de frecuencia en el sistema. Esto demuestra la correlación que existe entre el comportamiento dinámico que puede tener la frecuencia del sistema bajo un evento de falla por desconexión de una central generadora y la acción del sistema de control de inercia virtual de los parques eólicos.

Independientemente del período de simulación y del evento de falla específico, mientras mayor sea el valor de la constante de inercia, menor será la pendiente de la gráfica de la frecuencia del sistema. Esto quiere decir que, a mayor constante de inercia, menor será la caída de frecuencia en el mismo intervalo de tiempo.

$\mathrm{Al}$ examinar los escenarios bajo la salida de la central termoeléctrica Punta Catalina 1, con $377 \mathrm{MW}$, se puede observar un gran impacto sobre la frecuencia del sistema debido a la alta potencia. La frecuencia mínima se obtiene para el P04 y alcanza los $58.756 \mathrm{~Hz}$, específicamente para el escenario sin inercia virtual ya que no se inyecta ninguna potencia activa para oponerse al cambio de frecuencia (respuesta inercial). Cuando se hace la transición del escenario sin inercia hacia inercia de 1s, también se obtiene para el P04 la mayor diferencia entre frecuencias mínimas, siendo este diferencial de $19 \mathrm{mHz}$. Las pequeñas variaciones de las frecuencias mínimas que alcanza el sistema se deben al gran impacto de la salida de 377 MW.

Si se analizan los escenarios bajo el disparo de Itabo 1, con $125 \mathrm{MW}$, la frecuencia mínima se obtiene para el P04 y alcanza los $59.635 \mathrm{~Hz}$, específicamente para el escenario sin inercia virtual. Es posible observar que cuando se comparan las distintas magnitudes de constantes de inercia, la diferencia entre las frecuencias mínimas varía entre un rango de $6 \mathrm{mHz}$ y $12 \mathrm{mHz}$. 
Es importante destacar las diferencias entre los escenarios de ambos eventos simulados (Punta Catalina 1 e Itabo 1). A simple vista se confirma que la caída de frecuencia es mucho mayor en todos los casos de disparo de Punta Catalina 1. La gran capacidad de esta central frente a Itabo causa que su disparo repentino provoque una mayor inestabilidad en el sistema y peores niveles de frecuencia. Esto le dificulta al control de inercia virtual (con capacidad máxima de $17.5 \mathrm{MW}$ ) la tarea de oponerse al disparo y mantener más estables los niveles de frecuencia con su aporte de potencia activa, es decir, la respuesta inercial es menos efectiva que en el caso de Itabo. Demostrando así que conforme aumenta la magnitud de la constante de inercia, aumenta también la potencia aportada, y se mejoran notablemente cada vez más los niveles de frecuencia.

Por último, se distingue que el P04 resulta ser el período más representativo para mostrar las variaciones del comportamiento de las frecuencias. Este período corresponde al bloque de demanda mínima, por ende, existe menos generación y menos demanda en el sistema, y esto implica una menor inercia colectiva del sistema. Por estas razones, este período permite que el aporte de potencia activa sea cada vez más significativo y se noten mayores diferencias entre las frecuencias de los distintos escenarios de inercias.

Estos resultados representan un impacto significativo en la estabilidad del SENI, ya que aumenta su oposición a grandes fallas y extiende el tiempo que puede resistir durante estos eventos, con mejores niveles de frecuencia. Permitiendo así una mayor estabilidad y menos probabilidad de un posible colapso total.

\section{Conclusiones}

$\mathrm{Al}$ ejecutar las simulaciones con distintos escenarios se observó la misma tendencia a la reducción del tiempo de caída de la frecuencia ante una falla, lo que equivale a un aumento en la estabilidad del SENI ante fallas en el sistema, estabilidad que puede aumentar al aplicar inercia virtual en más plantas de generación basadas en tecnologías de fuentes 
renovables ya que en este análisis solo se contemplaron los 17.5 MW adicionales que serían capaces de inyectar los parques administrados por EGE Haina durante una falla.

Entre las tecnologías para la implementación de la inercial virtual que se estudiaron, la vertiente que se adaptó mejor al alcance de este proyecto fue la de ańadir una programación a la electrónica de potencia en el inversor del aerogenerador Full Converter para entregar potencia activa durante una falla en el SENI y con esto reducir el diferencial de frecuencia en el tiempo. En el caso del $D F I G$ la potencia activa se extrae del eje porque el inversor no trabaja con la potencia nominal del generador, por lo que hay que reducir la potencia nominal entregada en proporción a la potencia que se deja como reserva. Por otro lado, aunque no se utilizó en este caso, el almacenamiento de energía puede ser una opción en el momento que se decida regular frecuencia con tecnologías de fuentes renovables en una línea futura.

Para el modelo dinámico se utilizó como base de su funcionamiento el $R O C O F$, para que este actúe según el cambio de la frecuencia eléctrica e inyecte la potencia activa necesaria a el sistema eléctrico.

El escenario que mostró una mayor estabilidad de frecuencia, independientemente de la falla, se obtuvo al elegir una constante de inercia de 5s y una inyección de potencia activa nominal por parte de los aerogeneradores de 17.5 MW, que varía de acuerdo con el diferencial de frecuencia eléctrica en el SENI en el momento de la falla, y donde se aprecia más esta diferencia se encuentra en el periodo P04 debido a que es el periodo de demanda mínima, hay una menor inercia por parte del sistema.

Los resultados obtenidos en las simulaciones con el método de inercia virtual propuesto demuestran tener un impacto positivo en el sistema eléctrico, ya que este presenta una mejora en el tiempo que puede durar el sistema durante un evento como los presentados en las simulaciones y ayuda a la firmeza del sistema a oponerse a grandes fallas. 
La ausencia de un marco regulatorio no permite crear una relación directa entre la inercia virtual y la inserción de tecnologías de energía eólica en la República Dominicana, pero al tomar como base los estatutos de otros países, la inercia virtual solo representa un nivel de todos los que se deben cumplir para servicios auxiliares en tecnologías de energía renovables. Es cierto que en un futuro escenario donde las energías renovables tengan una mayor participación en la matriz energética, la inercia virtual agregaría robustez y confiabilidad al SENI, pero habría deficiencias en lo que respecta a regulación de voltaje y regulación de reactivos.

\section{Agradecimientos}

Se agradece a la empresa generadora de electricidad Haina (EGE Haina), por las informaciones suministradas durante el proceso de desarrollo del proyecto y las simulaciones realizadas en el software DIgSILENT.

\section{Referencias}

Agencia Internacional de Energías Renovables [IRENA]. (2017). Prospectivas de Energías Renovables: República Dominicana, REmap 2030. Abu Dhabi, www.irena.org/remap.Balali, M. H., Nouri, N., Omrani, E., Nasiri, A., \& Otieno, W. (2017). An overview of the environmental, economic, and material developments of the solar and wind sources coupled with the energy storage systems. International Journal of Energy Research, 41(14), 1948-1962. Disponible en: https://doi.org/10.1002/er.3755

Bada, C., Riva, L., \& Alvez, P. (2016). Efectos de la generaci'on e'olica frente al manejo de contingencias en el sistema interconectado uruguayo. (Tesis de Ingeniero), Universidad de la República, Montevideo. Retrieved from https://iie.fing.edu.uy/publicaciones/2016/ ABR16/ABR16.pdf

Deepak, C. M., Vijayakumari, A., \& Mohanrajan, S. R. (2017, 19-20 May 2017). Virtual inertia control for transient active power support from DFIG based wind electric system. Paper presented at the 2017 
2nd IEEE International Conference on Recent Trends in Electronics, Information \& Communication Technology (RTEICT). Disponible en https://doi.org/10.1109/RTEICT.2017.8256710

DIgSILENT. (2019). PowerFactory Applications, [en línea]. Disponible en: https://www.digsilent.de/en/powerfactory.html

European Commission. (2018). Fossil CO2 emissions of all world countries, 2018 report, [en línea]. Recuperado en 2 de octubre de 2019. Disponible en: https://edgar.jrc.ec.europa.eu/overview. php?v=booklet 2018

Gómez Molina, N., \& Rivera Rodríguez, S. R. (2017). Frequency regulation in power systems that integrate wind energy sources through a pi controller and inertial emulation. Ingeniería Solidaria, 13(23), 7-28. https://doi.org/10.16925/in.v23i13.1981Gonzalez-Longatt, F. M. (2015). Activation Schemes of Synthetic Inertia Controller on Full Converter Wind Turbine (Type 4). Paper presented at the 2015 IEEE Power \& Energy Society General Meeting. Disponible en https://doi.org/10.1109/PESGM.2015.7286430

Gonzalez-Longatt, F. M. (2016). Impact of emulated inertia from wind power on under-frequency protection schemes of future power systems. Journal of Modern Power Systems and Clean Energy, 4(2), 211-218. c10.1007/s40565-015-0143-x

Khaleghi Kerahroudi, S., M. Alamuti, M., Li, F., Taylor, G., \& Bradley, M. (2014). Application and Requirement of DIgSILENT PowerFactory to MATLAB/Simulink Interface. In F. M. Gonzalez-Longatt \& J. Luis Rueda (Eds.), PowerFactory Applications for Power System Analysis (pp. 297-322). Cham: Springer International Publishing. https://doi.org/10.1007/978-3-319-12958-7_13

Marabolí Muñoz, V. E. (2019). Análisis de la incorporación de capacidad de respuesta inercial en centrales fotovoltaicas mediante almacenamiento por baterías (BESS). Disponible en http://repositorio. uchile.cl/handle/2250/170284

Nuñez, J., Cepeda, J., \& Salazar,. (2015). Comparación Técnica entre los Programas de Simulación de Sistemas de Potencia DIgSILENT PowerFactory y PSS/E. (11). Disponible en https://bibdigital.epn. edu.ec/handle/15000/10316 
Salinas Sánchez, C. (2016).Diseño de esquemas de control para respuesta inercial en generadores de inducción doblemente alimentado (DFIG). Disponible en http://repositorio.uchile.cl/handle/2250/140978

SIEMENS. (2019). $\mathrm{PSS}^{\circ} \mathrm{E}$ - high-performance transmission planning and analysis software. Retrieved from https://new.siemens.com/global/ en/products/energy/services/transmission-distribution-smart-grid/ consulting-and-planning/pss-software/pss-e.html

Tamrakar, U., Shrestha, D., Maharjan, M., Bhattarai, P. B., Hansen, M. T., \& Tonkoski, R. (2017). Virtual Inertia: Current Trends and Future Directions. Applied Sciences, 7(7), 654. MDPI AG. Retrieved from https://doi.org/10.3390/app7070654

Tielens, P., Van Hertem, D. J. R., \& Reviews, S. E. (2016). The relevance of inertia in power systems. Renewable and Sustainable Energy Reviews, 55, 999-1009. https://doi.org/10.1016/j.rser.2015.11.016

United States Agency for International Development. (2017). Greenhouse Gas Emissions in Dominican Republic. In. Republica Dominicana: United States Agency for International Development (USAID). https://www.climatelinks.org/sites/default/files/asset/document/2017_USAID_GHG\%20Emissions\%20Factsheet_Dominican\%20Republic.pdf

León Viltre, Lesyani T., Hernández Amador, Leonardo, \& Aybar Mejia, Miguel. (2019). Island detection methods in doubly fed induction generators. Ingeniería Energética, 40(2), 128-137. Recuperado en 2 de octubre de 2019, de http://scielo.sld.cu/scielo.php?script=sci_ arttext\&pid=S1815-59012019000200128\&lng=es\&tlng=en. 